\title{
Effects of Ruthenium Red on the Cellular Functions and Ultrastructure in Intact Ferret Ventricular Muscles
}

\author{
Etsuko TANAKA, Makoto KAWAI, Satoshi KURIHARA, Yasuaki HOTTA*, and Tsuyoshi SOJl* \\ Department of Physiology, The Jikei University School of Medicine, Minato-ku, Tokyo, 105 Japan; and \\ *Department of Anatomy, Nagoya City University Medical School, Nagoya, 467 Japan
}

\begin{abstract}
The effects of ruthenium red (RR) on the cellular functions (intracellular $\mathrm{Ca}^{2+}$ handling and contraction) and permeation of the dye through the cell membrane were investigated in intact ferret papillary muscles. The intracellular $\mathrm{Ca}^{2+}$ concentration $\left(\left[\mathrm{Ca}^{2+}\right]_{\mathrm{i}}\right)$, measured using aequorin, was simultaneously recorded with tension. The permeation of the dye through the cell membrane was studied with electronmicroscopy. The preparation was continuously stimulated at $0.2 \mathrm{~Hz}$ and treated with $50 \mu \mathrm{M}$ RR at $30^{\circ} \mathrm{C}$. $\left[\mathrm{Ca}^{2+}\right]_{\mathrm{i}}$ was increased by electrical stimulation $(0.07$ and $2 \mathrm{~Hz}$ ) and rapid cooling (from 30 to $\left.4^{\circ} \mathrm{C}\right)(\mathrm{RC})$. In electrical stimulation, RR time-dependently decreased the peak light of aequorin without a significant change in the time course at $30^{\circ} \mathrm{C}$. However, in $\mathrm{RC}$, treatment with $\mathrm{RR}$ for about $100 \mathrm{~min}$ significantly prolonged the decay
\end{abstract}

time of the light signal and increased the peak light. The peak tension in $\mathrm{RC}$ was decreased after treatment with $\mathrm{RR}$ for a longer time. The pCa-tension relation of skinned preparations was significantly shifted to the right by $50 \mu \mathrm{M} R R$. In the RR $(50 \mu \mathrm{M})$-treated specimens, mitochondrial outer membranes were darkly stained if $\mathrm{OsO}_{4}$ was used for fixation. Even though the specimen treated with $500 \mu \mathrm{M}$ RR was fixed without $\mathrm{OsO}_{4}$ and electron staining, the matrices of mitochondria became electron dense. We concluded that RR could penetrate into intact mammalian cardiac myocytes, and that RR inhibits the release of $\mathrm{Ca}^{2+}$ from the sarcoplasmic reticulum in electrical stimulation, inhibits mitochondrial $\mathrm{Ca}^{2+}$ uptake, and decreases the $\mathrm{Ca}^{2+}$ sensitivity of the myofilaments. [Japanese Journal of Physiology, 47, 273-281, 1997]

Key words: ruthenium red, sarcoplasmic reticulum, mitochondria, $\mathrm{Ca}^{2+}$ sensitivity, cardiac muscle.

$\mathrm{R}$ [1-3] which has various effects on excitation-contraction coupling in skeletal and cardiac muscles: modification of the $\mathrm{Ca}^{2+}$ release from the sarcoplasmic reticulum (SR) [4-8], inhibition of mitochondrial $\mathrm{Ca}^{2+}$ uptake [9-11], alteration of the $\mathrm{Ca}^{2+}$ sensitivity of the myofilaments $[6,12,13]$, and changes in the electrical properties of the cell membrane [12].

However, the permeation of RR through the cell membrane of intact muscle is considered difficult because RR is a highly charged molecule. Therefore, the effects of RR have been studied using isolated SR and mitochondria or by the injection of RR into intact muscle fibers.
In this study, we investigated the effects of RR on the $\mathrm{Ca}^{2+}$ release from the SR, mitochondrial $\mathrm{Ca}^{2+}$ uptake, and the $\mathrm{Ca}^{2+}$ sensitivity of the contractile elements in intact and skinned papillary muscles of ferrets. For this purpose, we used the aequorin method to measure intracellular $\mathrm{Ca}^{2+}$ concentration $\left(\left[\mathrm{Ca}^{2+}\right]_{i}\right)$ change for identification of the changes in the SR and mitochondrial functions. Triton X-100-treated skinned preparation was also employed to observe whether or not $\mathrm{RR}$ alters the $\mathrm{Ca}^{2+}$ sensitivity of the contractile elements. Furthermore, we observed the morphological changes of the mitochondrial membrane using electronmicroscopy to confirm the permeation of RR through the cell membrane.

Received on September 24, 1996; accepted on February 17, 1997

Correspondence should be addressed to: Etsuko Tanaka, Department of Physiology, The Jikei University School of Medicine, 3-25-8 Nishishinbashi, Minato-ku, Tokyo, 105 Japan. Tel: +81-3-3433-1111 ext. 2221, Fax: +81-3-3433-1279 
A portion of these results was presented at the Physiological Society of Japan and published in abstract form [14].

\section{METHODS}

Preparations for the experiments using the aequorin method. Ferrets (body weight 600 $1,200 \mathrm{~g}$ ) were anaesthetized by intraperitoneal injection of pentobarbitone $\left(150 \mathrm{mg} \cdot \mathrm{kg}^{-1}\right)$ and the hearts were quickly removed. The right ventricular wall was opened and papillary muscles were dissected out in normal Tyrode's solution at $30^{\circ} \mathrm{C}$. The diameter of the preparation was $0.4-1.0 \mathrm{~mm}(0.7 \pm 0.04 \mathrm{~mm}$, mean \pm standard error of the mean (SEM), $n=9$ ) and the length of the preparation was $3.2-5.2 \mathrm{~mm} \mathrm{(4.0 \pm}$ $0.7 \mathrm{~mm}, n=9$ ). The preparation was horizontally mounted in a muscle chamber with a pair of platinum black wires for electrical stimulation. One end of the preparation was connected to a fixed hook and the other end was attached to the arm of a tension transducer (BG-10, Kulite, NJ, USA). The preparation was stimulated by a square pulse with a $5 \mathrm{~ms}$ duration at 1.5 -fold the threshold unless otherwise mentioned. Before starting the experiment, the preparation was slowly stretched to develop maximal tension of the preparation $\left(L_{\max }\right)$.

Solutions. The composition of the normal Tyrode's solution used for dissection and aequorin injection was as follows $(\mathrm{mM}): \mathrm{Na}^{+}, 135 ; \mathrm{K}^{+}, 5 ; \mathrm{Ca}^{2+}, 2$; $\mathrm{Mg}^{2+}, 1 ; \mathrm{Cl}^{-}, 102 ; \mathrm{HCO}_{3}{ }^{-}, 20 ; \mathrm{HPO}_{4}{ }^{2-}, 1 ; \mathrm{SO}_{4}{ }^{2-}, 1$; acetate, 20; glucose, 10; and insulin, 5 units $\cdot l^{-1}$. The $\mathrm{pH}$ was $7.3-7.4$ at $30^{\circ} \mathrm{C}$ equilibrated with $5 \% \mathrm{CO}_{2}+$ $95 \% \quad \mathrm{O}_{2}$. The Tyrode's solution buffered with $\mathrm{N}$-2-hydroxyethyl-piperazine- $\mathrm{N}$ '-2-ethanesulfonic acid (HEPES) used in the experiments (HEPES-Tyrode's solution) had the following composition (mM): $\mathrm{Na}^{+}, 128 ; \mathrm{K}^{+}, 5 ; \mathrm{Ca}^{2+}, 2 ; \mathrm{Mg}^{2+}, 1 ; \mathrm{Cl}^{-}, 117 ; \mathrm{SO}_{4}{ }^{2-}$, 1; acetate, 20; glucose, 10; HEPES, 5; and insulin, 5 units $\cdot l^{-1}$. The $\mathrm{pH}$ was adjusted to $7.3-7.4$ with $\mathrm{NaOH}$ at $30^{\circ} \mathrm{C}$ and was equilibrated with $100 \% \mathrm{O}_{2}$.

Aequorin injection and light detection. Aequorin (purchased from Dr. J. R. Blinks) was dissolved in the solution containing $200-300 \mathrm{mM} \mathrm{KCl}$ and $10 \mathrm{mM}$ HEPES at $\mathrm{pH} 7.0$ with a final concentration of $100-150 \mu \mathrm{M}$. For aequorin injection, glass micro-pipettes with a resistance of $30-50 \mathrm{M} \Omega$ were prepared and filled with aequorin. Aequorin was injected into 100-200 superficial cells of each preparation by applying high-pressure $\left(4-7 \mathrm{~kg} \cdot \mathrm{cm}^{-2}\right)$ nitrogen gas. Aequorin light signals were detected with a photomultiplier (EMI 9789A, Ruislip, UK) placed just above the preparation and were simultaneously recorded with tension. Light signals in rapid cooling (RC) were recorded through a $1 \mathrm{~Hz}$ low-pass filter.

We checked the direct effect of RR on aequorin luminescence in a cuvette. The intensity of aequorin luminescence was decreased to $89 \%$ of the control by solution containing $100 \mu \mathrm{M}$ RR and to $67 \%$ of the control by $500 \mu \mathrm{M} R$ R. In these experiments, we extracellulary applied $50 \mu \mathrm{M} R \mathrm{RR}$ to measure the change in $\left[\mathrm{Ca}^{2+}\right]_{\mathrm{i}}$ and tension. Therefore, the intracellular $\mathrm{RR}$ concentration is expected to be much lower than $50 \mu \mathrm{M}$ and the direct effect of RR on aequorin luminescence should be much less than that measured in a cuvette. Thus, the inhibition of aequorin light signal by $\operatorname{RR}(50 \mu \mathrm{M})$ is less than $10 \%$ in this experiment.

Protocols for the experiments using the aequorin method. We measured intracellular $\mathrm{Ca}^{2+}$ transients induced by electrical stimulation to observe whether or not RR could penetrate into the myoplasm and inhibit $\mathrm{Ca}^{2+}$ release from the SR. For loading $\mathrm{RR}$ into the cells, the preparation was treated with $50 \mu \mathrm{M}$ $\mathrm{RR}$ while stimulating at $0.2 \mathrm{~Hz}$ for 80 to $190 \mathrm{~min}$ at $30^{\circ} \mathrm{C}$. After the treatment, the light signals and tension induced by electrical stimulation at 0.07 and $2 \mathrm{~Hz}$ were measured to confirm that $\mathrm{RR}$ could inhibit $\mathrm{Ca}^{2+}$ release from the SR induced by electrical stimulation.

In the second protocol, $\mathrm{Ca}^{2+}$ was directly released from the SR by rapid cooling (RC), which releases $\mathrm{Ca}^{2+}$ from the SR by a different mode from that by electrical stimulation [15]. After repetitive electrical stimulation ( $2 \mathrm{~Hz}$ for $5 \mathrm{~min}$ ), which was used for loading the SR with $\mathrm{Ca}^{2+}$, the temperature of the solution was rapidly $(<1 \mathrm{~s})$ lowered from 30 to $4^{\circ} \mathrm{C}$ [16-18]. After recording the control $\mathrm{RC}$, the preparation was treated with $50 \mu \mathrm{M} R \mathrm{RR}$ while applying electrical stimulation at $0.2 \mathrm{~Hz}$ for 80 to $190 \mathrm{~min}$ at $30^{\circ} \mathrm{C}$. Then, the light signals and tension induced by RC were simultaneously recorded. We measured the peak light and peak tension simultaneously to investigate the $\mathrm{Ca}^{2+}$ sensitivity of the myofilaments. In $\mathrm{RC}$, we also measured the half decay time of the light signal to observe whether $\mathrm{Ca}^{2+}$ removal by mitochondria is influenced by RR [19].

Skinned preparations. Thin trabeculae and papillary muscles were dissected from the right ventricle of ferrets. The papillary muscles were cut into a small bundle along the longitudinal axis in a relaxing solution. The preparations were treated with the relaxing solution containing $1 \%$ Triton $\mathrm{X}-100$ for $60 \mathrm{~min}$. After treatment of the preparations with Triton X-100, the preparations were washed with the relaxing solution without Triton X-100. The preparations were then immersed in a relaxing solution containing $50 \%$ glycerol and kept at $-4^{\circ} \mathrm{C}$ before use. The diameter of the 
preparation was $150-250 \mu \mathrm{m}(214 \pm 38 \mu \mathrm{m}, n=7)$. The length was $2.0-5.5 \mathrm{~mm}(4.1 \pm 1.1 \mathrm{~mm}, n=7)$. Both ends of the preparation were tied with silk monofilaments, and then the preparation was transferred carefully to a muscle chamber. One end of the preparation was fixed to a tungsten wire ( $100 \mu \mathrm{m}$ in diameter) extending from the fixed arm and the other end was connected to the arm of the tension transducer (BG-10, Kulite). The sarcomere length of the preparation was adjusted to $2.4 \mu \mathrm{m}$ by measuring the laser diffraction lines.

Solutions for skinned preparations. The composition of the relaxing solution was as follows $(\mathrm{mM}): \mathrm{K}$ methanesulfonate, $74 ; \mathrm{ATP}, 4.6 ; \mathrm{Mg}$ methanesulfonate, 5.2; EGTA, 10; PIPES (piperazine$\mathrm{N}, \mathrm{N}^{\prime}$-bis-2-ethanesulfonic acid), 20; creatine phosphate, 10; and creatine phosphokinase, $10 \mathrm{IU} / \mathrm{ml}$. The $\mathrm{pH}$ was adjusted to 7.0 using $\mathrm{KOH}$. The $5.0 \mathrm{pCa}$ solution was composed of the following $(\mathrm{mM})$ : $\mathrm{K}$ methanesulfonate, 55; ATP, 4.6; Mg methanesulfonate, 4.9; $\mathrm{Ca}$ methanesulfonate, 9.6; EGTA, 10; PIPES, 20; creatine phosphate, 10; and creatine phosphokinase, $10 \mathrm{IU} / \mathrm{ml}$. The $\mathrm{pH}$ was adjusted to 7.0 using $\mathrm{KOH}$. The free $\mathrm{Ca}^{2+}$ concentration of the solution was calculated using the binding constants of each ion for each ligand. The calculated dissociation constant of EGTA for $\mathrm{Ca}^{2+}$ was $407 \mathrm{nM}$. The concentration of free $\mathrm{Mg}^{2+}$ and $\mathrm{Mg}$-ATP were kept at 1.0 and $3.5 \mathrm{mM}$, respectively. Ionic strength was maintained at $0.2 \mathrm{M}$. The solutions of differing $\mathrm{pCa}$ were made by mixing the relaxing solution and the solution with pCa 5.0. The temperature of the solution was kept at $20 \pm 1^{\circ} \mathrm{C}$ throughout the experiment.

Experimental procedures and protocol for skinned preparations. Contraction of skinned fibers was induced by an activating solution at different pCa by quickly moving the muscle chamber. The solution was changed within $1 \mathrm{~s}$. The preparation was activated with the solution with $\mathrm{pCa} 5.0$ and then washed with the relaxing solution. The pCa-tension relation was measured by applying solutions at different $\mathrm{pCa}$ in an additive manner. Then, the preparation was washed with the relaxing solution. The preparation was treated with relaxing solution containing $R R$ $(50 \mu \mathrm{M})$ for $10 \mathrm{~min}$. Thereafter, the preparation was activated by applying a solution with a different $\mathrm{pCa}$ as in the control. The maximal tension induced by the solution with pCa 5.0 was slightly decreased $(8 \pm 4 \%$, $n=7$ ) during the course of the experiment. The measured tension induced by the solution at each $\mathrm{pCa}$, with or without RR, was normalized to that of the maximal tension at $\mathrm{pCa} 5.0$. The measured points were fitted with Hill's equation: $F=F_{\max }\left[\mathrm{Ca}^{2+}\right]_{i}^{n} /$
$K_{1 / 2}{ }^{n}+\left[\mathrm{Ca}^{2+}\right]_{i}^{n}$, where $F_{\max }$ is the maximal force, $K_{1 / 2}$ is $\left[\mathrm{Ca}^{2+}\right]_{i}$ at half $F_{\max }$, and $n$ is the Hill coefficient.

Electronmicroscopic study. We used three different fixation and electron staining methods (glutaraldehyde $+\mathrm{OsO}_{4}+$ uranyl acetate + lead citrate, glutaraldehyde+uranyl acetate +lead citrate, glutaraldehyde only). The reason why we chose these fixation methods was to show that the high electron density images were not due to the artifact of the fixation methods.

Three papillary muscles were used for the electronmicroscopic study. The diameter of the specimens was $0.8-1.2 \mathrm{~mm}(0.92 \pm 0.06 \mathrm{~mm}, n=3)$ and the length was $2.6-4.4 \mathrm{~mm}(3.33 \pm 0.55 \mathrm{~mm}, n=3)$. Papillary muscles were dissected out from the right ventricle as in the case of the aequorin experiment, and were mounted in the same muscle chamber. Muscle length was adjusted to $L_{\max }$, which produced maximal twitch tension of the preparation.

The first specimen was used for the control and immersed in normal Tyrode's solution during the application of electrical stimulation at $0.2 \mathrm{~Hz}$ for the same period as the second specimen. The second specimen was immersed in Tyrode's solution containing $50 \mu \mathrm{M}$ $\mathrm{RR}$ for $80 \mathrm{~min}$ at $30^{\circ} \mathrm{C}$ during application of electrical stimulation at $0.2 \mathrm{~Hz}$. Both specimens (the control and RR-treated specimen) were fixed with $0.1 \mathrm{M}$ phosphate-buffered ( $\mathrm{pH} 7.4$ ) solution containing $2.5 \%$ glutaraldehyde for $12 \mathrm{~h}$. Then, the specimens were rinsed with the same solution and post-fixed with $0.1 \mathrm{M}$ phosphate-buffered ( $\mathrm{pH} 7.4) 1 \% \mathrm{OsO}_{4}$ solution. After fixation, the specimens were dehydrated with a graded series of ethanol. Then, the specimens were further treated with QY-1 ( $n$-butyl glycidyl ether) and embedded in Epon 812 resin. If RR penetrated into the myoplasm, the mitochondrial membranes were stained, because RR is known to further enhance the electron density of the membrane which is increased by $\mathrm{OsO}_{4}$. Fifteen parts in the core and surface of the specimen were randomly selected. The number of mitochondria with a darkly stained membrane was totaled, and the number of darkly-stained mitochondria in the control and RR-treated specimens was compared. A third preparation was treated with $500 \mu \mathrm{M} R \mathrm{RR}$ for $110 \mathrm{~min}$ at $30^{\circ} \mathrm{C}$ during application of electrical stimulation at $0.2 \mathrm{~Hz}$. After that, the specimen was simply fixed with $0.1 \mathrm{M}$ phosphate solution containing $2.5 \%$ glutaraldehyde without $\mathrm{OsO}_{4}$. The specimen was divided into two pieces. One of the two pieces was stained with uranyl acetate and lead citrate. The other piece was not stained.

Chemicals. All chemicals used in this experiment were of analytical grade and purchased from the 
following companies: ATPNa $\mathrm{Na}_{2}$, Boehringer Mannheim, Germany; methanesulfonic acid and $\mathrm{Ca}$ methanesulfonate, Tokyo Kasei Co. Ltd., Tokyo, Japan; creatine phosphate, EGTA and ruthenium red, Nacalai Tesque Inc., Kyoto, Japan; creatine phosphokinase, Sigma Chemical Co., St. Louis, MO, USA; Triton X100, Wako Pure Chemical Ind. Ltd., Osaka, Japan.

Statistics. Measured values were expressed as the mean \pm standard error of the mean (SEM) in the text and figures. Student's $t$-test (two-tailed for unpaired samples) was used to test for significant differences in the measured values. A significant difference was verified at $p<0.05 . \chi^{2}$ analysis was also used to test the independence of the measured values.

\section{RESULTS}

\section{Effect of $\mathrm{RR}$ on $\mathrm{Ca}^{2+}$ release from $\mathrm{SR}$ with electrical stimulation}

The peak of the aequorin light signal induced by electrical stimulation at $0.07 \mathrm{~Hz}$ was decreased 120 min after treatment with RR (Fig. 1A). The peak of the light signal induced by electrical stimulation at
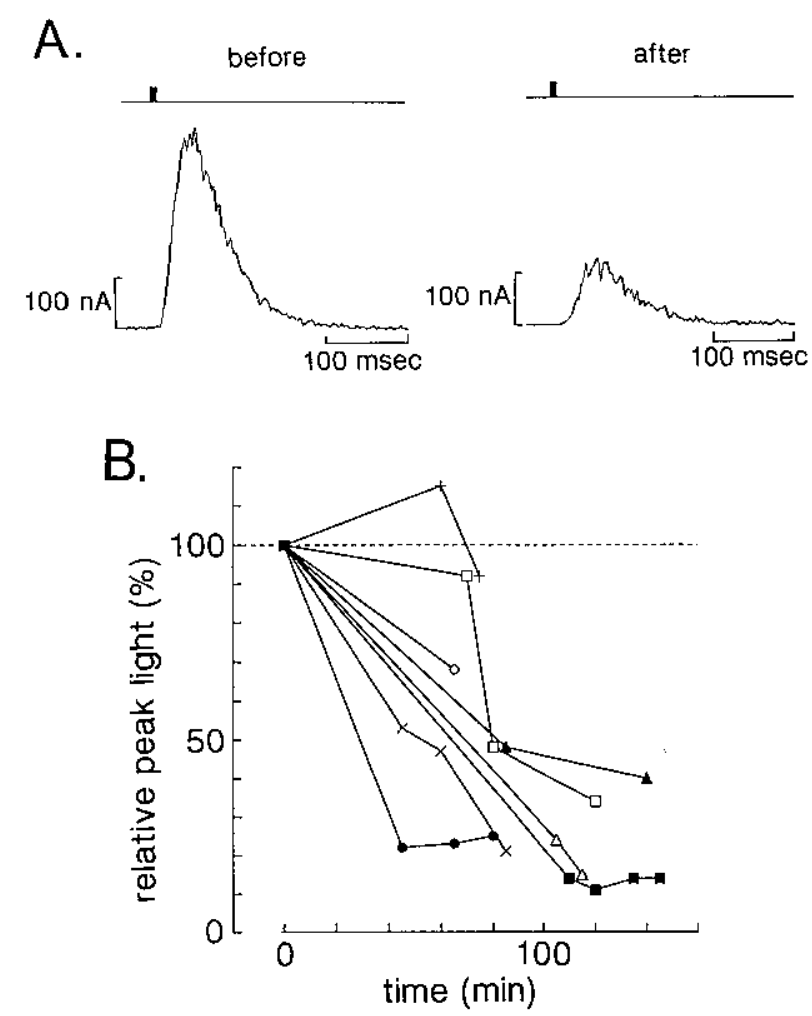

Fig. 1. Effects of ruthenium red (RR) on $\mathrm{Ca}^{2+}$ signals induced by electrical stimulation (ES). A: The aequorin light signals induced by ES at $0.07 \mathrm{~Hz}$ before (left panel) and after (middle panel) treatment with $50 \mu \mathrm{M}$ RR for $120 \mathrm{~min}$. The change in the relative peak light induced with ES at $2 \mathrm{~Hz}$ was plotted against the RR treatment time $(n=8)$ (B).
$2 \mathrm{~Hz}$, which was applied immediately before $\mathrm{RC}$, decreased in accordance with the treatment time in all eight preparations tested (Fig. 1B).

\section{Effects of RR on the aequorin light signal and tension change induced by RC}

The peak of the aequorin light signal induced by $\mathrm{RC}$ increased in all nine preparations tested when the RR treatment period was prolonged. This increase was enhanced by an $R R$ treatment length of longer than $100 \mathrm{~min}$ (Figs. 2A and $3 \mathrm{~A}$ ). In parallel with the increase in the peak light, the decay time of the light signal was significantly prolonged (Figs. $2 \mathrm{~B}$ and $3 \mathrm{C}$ ).

The peak of the developed tension in $\mathrm{RC}$ increased in parallel with the increase in light signal, which was observed only for a limited time $(60-140 \mathrm{~min})$ of treatment with RR. However, if the treatment period was longer than the limited time, the peak tension decreased (Figs. 2A and 3B).

\section{Effect of RR on the pCa-tension relation in skinned preparations}

Figure 4 shows the pCa-tension relation in the skinned preparations in the presence or absence of $50 \mu \mathrm{M} \mathrm{RR}(n=7)$. This relation, measured by applying solutions with different $\mathrm{pCa}$ in an additive manner, was significantly shifted to the right by RR. The change in $\mathrm{pCa}_{50}$ required for half maximal activation was $0.08 \mathrm{pCa}$ unit $(p<0.01)$. The Hill coefficients in the presence or absence of $50 \mu \mathrm{M} R \mathrm{RR}$ were $3.84 \pm 0.13$ and $4.07 \pm 0.13$, respectively (no significant difference).

\section{Evidence of the permeation of RR through the cell membrane of intact living papillary muscles}

In the control specimens, darkly stained (electron dense) mitochondrial membranes were not observed in any of the specimens tested. However, some of the mitochondrial membranes of the RR-treated specimens were darkly stained as shown in Fig. 5A. In the RR-treated specimens, 35 mitochondria showed darkly stained membrane among the 465 mitochondria randomly selected in the 15 parts of the surface area of the specimen (shown in Fig. 5B, inset). In contrast, the control specimen did not contain mitochondria with a darkly stained outer membrane, which was confirmed in all 553 mitochondria in the 15 parts of the surface area $(p<0.01)$. The percentage of mitochondria with a darkly stained outer membrane in the 15 parts of the surface area was larger than that of the core $(7.5 \%$ vs. $1.6 \%, p<0.01)$ (Fig. $5 \mathrm{~B})$.

A darkly stained mitochondrial matrix was ob- 


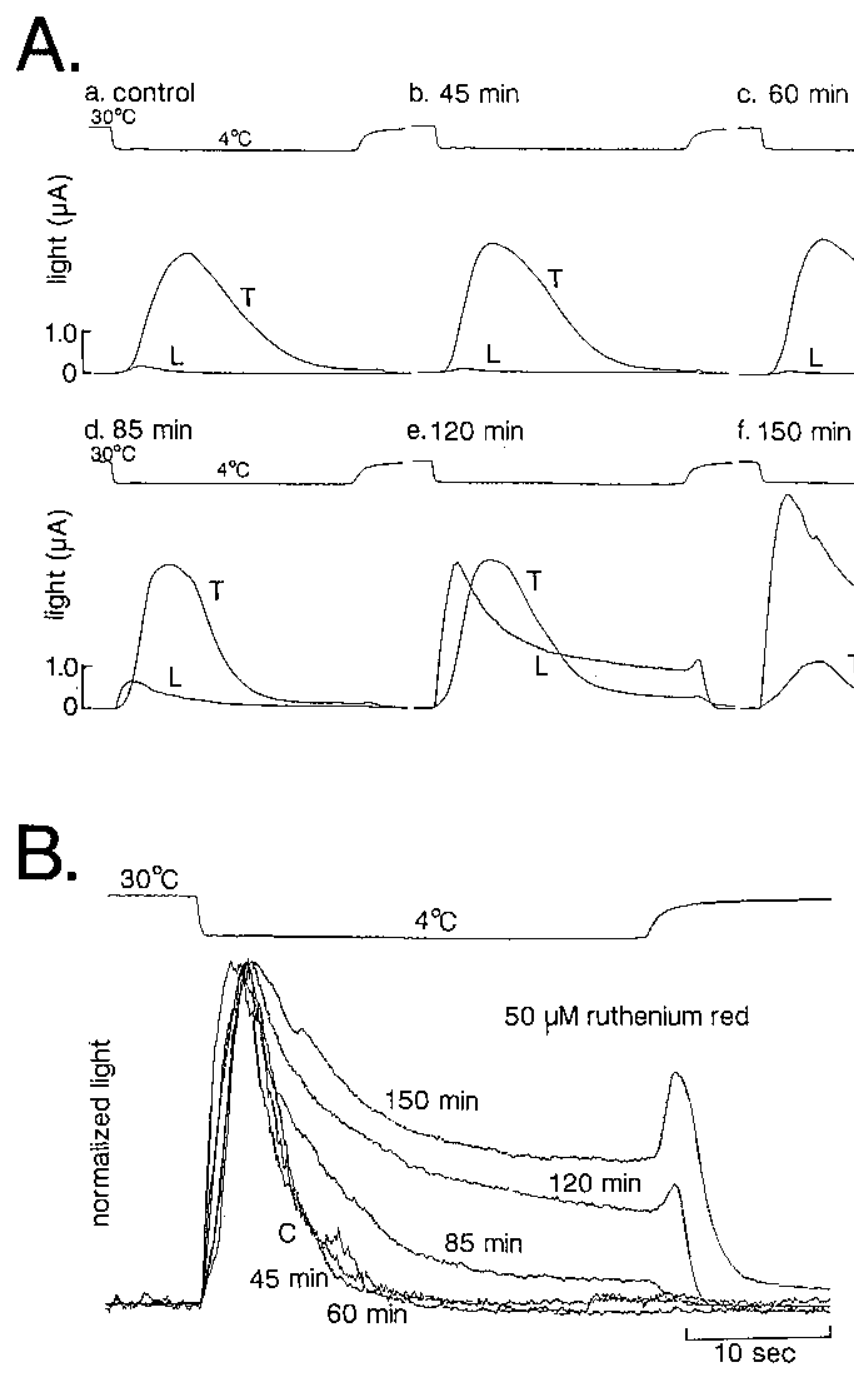

served in the specimen fixed without $\mathrm{OsO}_{4} 110 \mathrm{~min}$ after immersion of the preparation in the solution containing $500 \mu \mathrm{M}$ RR (Fig. 6A). Furthermore, a darkly stained mitochondrial matrix was also observed in the specimen which was not treated with $\mathrm{OsO}_{4}$ or other electron staining agents (uranyl acetate and lead citrate) (Fig. 6B).

No darkly stained membrane was recognized in the SR or in other organella in all preparations examined.

\section{DISCUSSION}

This study shows that RR influences the intracellular $\mathrm{Ca}^{2+}$ handling and tension development in intact ferret papillary muscles, suggesting that RR can permeate through the intact cell membrane. The permeation of RR was confirmed by an electronmicroscopic study which showed darkly stained mitochondrial membranes, one of the most possible effective sites of RR, in the RR-treated preparations.

Effects of RR on $\mathrm{Ca}^{2+}$ handling. Two differ-
Fig. 2. Effects of $\mathrm{RR}$ on $\mathrm{Ca}^{2+}$ release from the SR by rapid cooling (RC) from 30 to $4^{\circ} \mathrm{C}$. The light signal $(L)$ and tension $(T)$ were recorded in the control and after RR treatment for $45,60,85,120$, and $150 \mathrm{~min}(\mathbf{A})$. Normalized light signals to match their peaks in the same experiment as shown in $\mathrm{A}$ are superimposed in $\mathbf{B}$.

ent changes in the peak of the $\mathrm{Ca}^{2+}$ signal were observed after treatment with RR: a decrease in the $\mathrm{Ca}^{2+}$ signal induced by electrical stimulation (Fig. 1A and $\mathrm{B}$ ) and an increase in the $\mathrm{Ca}^{2+}$ signal in RC (Figs. 2A and $3 \mathrm{~A}$ ). The decrease in the $\mathrm{Ca}^{2+}$ signal induced by electrical stimulation was not due to the direct effect of RR on aequorin because the inhibition of aequorin luminescence by RR tested in a cuvette could not explain the larger decrease of the light signal with electrical stimulation (see METHODS section). A possible explanation for the decrease in the $\mathrm{Ca}^{2+}$ signal is the inhibition of $\mathrm{Ca}^{2+}$ release from the SR [4-8], and a reduction of the open probability of the $\mathrm{Ca}^{2+}$ release channels of the SR by RR has been directly shown [20]. On the other hand, RR increased the amplitude of the light signal induced by $\mathrm{RC}$, which is considered to directly release $\mathrm{Ca}^{2+}$ from the SR. The $\mathrm{RC}$-induced $\mathrm{Ca}^{2+}$ release mechanism is probably different from the $\mathrm{Ca}^{2+}$ release induced by $\mathrm{Ca}^{2+}$, the action potential and caffeine $[15,18]$. A large $\mathrm{Ca}^{2+}$ signal induced by $\mathrm{RC}$ in the presence of $\mathrm{RR}$ suggests that the $\mathrm{SR} \mathrm{Ca}^{2+}$ content is increased by $\mathrm{RR}$ and this accumulated $\mathrm{Ca}^{2+}$ can be released by $\mathrm{RC}$.

The increase in the $\mathrm{Ca}^{2+}$ signal by $\mathrm{RR}$ during $\mathrm{RC}$ was accompanied by a considerably slower time course. Since the $\mathrm{Ca}^{2+}$ released by RC is mainly removed by mitochondrial $\mathrm{Ca}^{2+}$ uniport from the myoplasm at a low temperature [19], the slower time course could be explained by the inhibition of the $\mathrm{Ca}^{2+}$ uptake by the mitochondrial $\mathrm{Ca}^{2+}$ uniport by $\mathrm{RR}$ [9-11, 21-23].

A recent study analyzing the effective component of $\mathrm{RR}$ in the inhibition of the mitochondrial $\mathrm{Ca}^{2+}$ uniport clarified that a derivative, $\mu-\mathrm{O}-\left[\left(\mathrm{HCO}_{2}\right)\left(\mathrm{NH}_{3}\right)_{4}\right.$ $\mathrm{Ru}]_{2} \mathrm{Cl}_{3}$, is the most effective agent contained in RR 

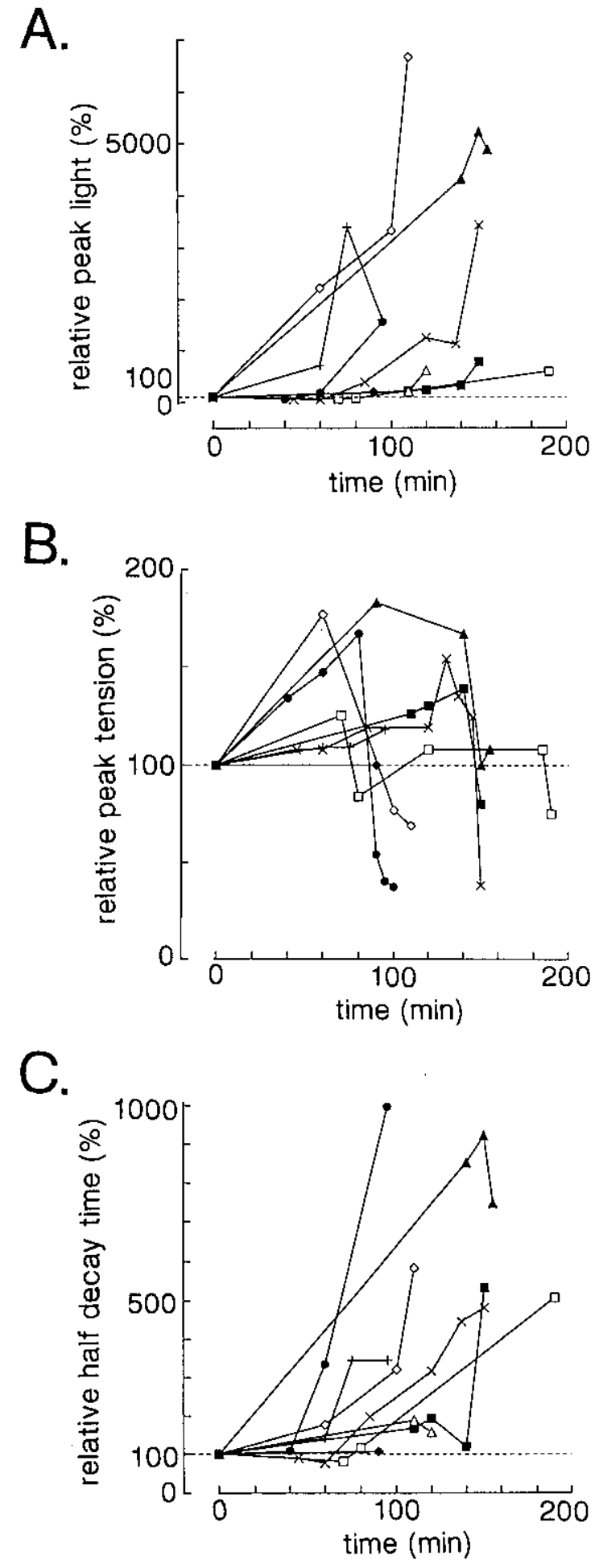

Fig. 3. Time-dependent changes of the relative peak light (A), relative peak tension (B), and relative half decay time of the light signals $(C)$ induced by $R C$ after RR treatment. In almost all of the cases tested, the peak and half decay time of the light signal increased gradually. However, tension gradually increased with $100 \mathrm{~min}$ to 140 min treatment and then abruptly decreased. Number of measurements was 9 .

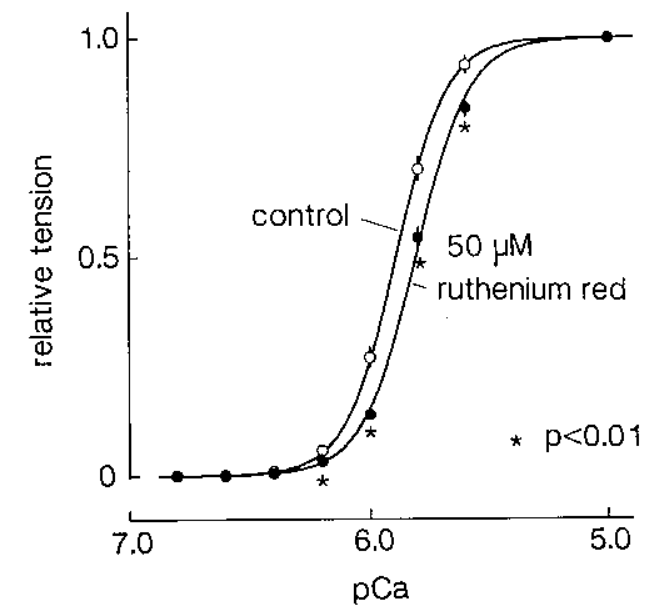

Fig. 4. pCa-tension relation measured in the skinned preparation in the presence or absence of $50 \mu \mathrm{M}$ RR. Each symbol indicates the mean value of relative tension \pm SEM (7 preparations). The curve fitted with the Hill equation was significantly shifted to the right by $50 \mu \mathrm{M} R R$ in the middle range of the $\mathrm{pCa}$-tension relation. The change in $\mathrm{pCa}_{50}$ was 0.08 unit $(p<0.01)$. The Hill coefficient in the presence or absence of RR was 3.84 and 4.07 , respectively (no significant change).

[24]. The RR used in this study is believed to have contained a significant amount of this agent because the expected change in $\mathrm{Ca}^{2+}$ handling did occur.

The inhibition of the $\mathrm{Ca}^{2+}$ signal in electrical stimulation appeared earlier compared to the increase of the $\mathrm{Ca}^{2+}$ signal during $\mathrm{RC}$ (Figs. $1 \mathrm{~B}$ and $3 \mathrm{~A}$ ). This fact can be explained by two mechanisms: the SR located at the surface area of the cell can be more effectively influenced by RR, and RR is more effective for the $\mathrm{Ca}^{2+}$ release mechanism in the SR than for the $\mathrm{Ca}^{2+}$ uniport of mitochondria.

Change in tension development during RC. $R R$ increased and then decreased the developed tension during $\mathrm{RC}$ as shown in Fig. 3B. The increase of tension observed in the earlier phase after RR treatment can be explained by the increased $\mathrm{Ca}^{2+}$ signal. The decrease in tension at the later phase of treatment was due to the $\mathrm{Ca}^{2+}$ desensitizing effect of RR, because $\mathrm{RR}$ shifted the $\mathrm{pCa}$-tension relation of the skinned preparation to the right (Fig. 4). This result also confirms the permeation of RR through the cell membrane of the preparation. The rightward shift in the pCa-tension relation in the skinned preparation is small compared to the large reduction of tension during $\mathrm{RC}$ in intact papillary muscles. One of the reasons for the apparently different change in tension in both intact and skinned preparations is the different experimental conditions, as noted below. The pCa-tension relation was measured at $20^{\circ} \mathrm{C}$ but tension during $\mathrm{RC}$ was measured at a temperature below $4^{\circ} \mathrm{C}$. Therefore, 
A.

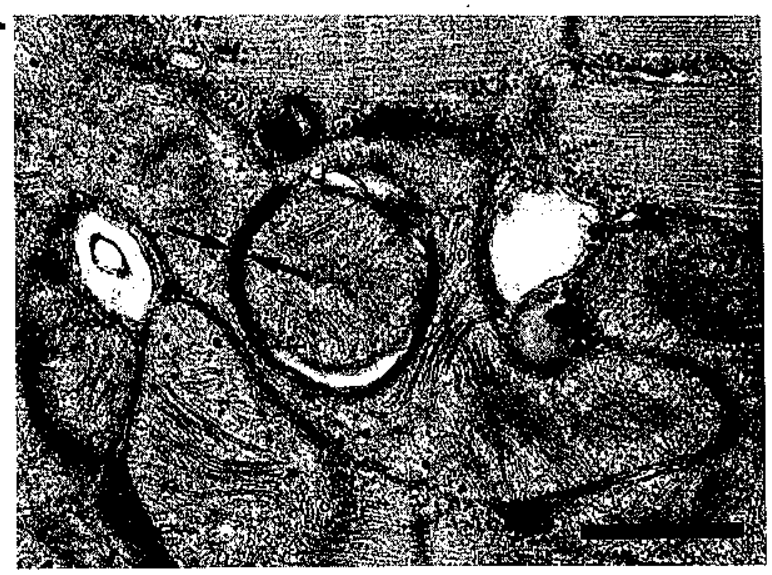

B.
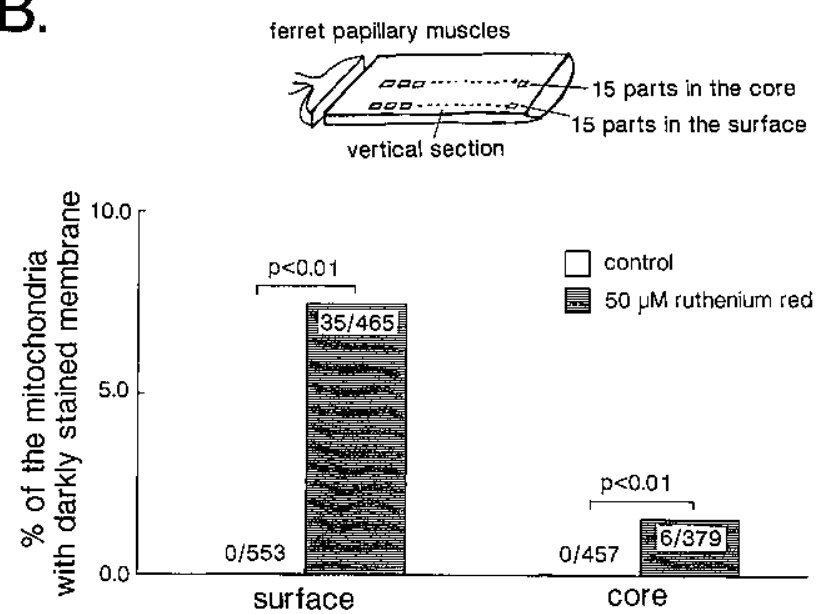

A.

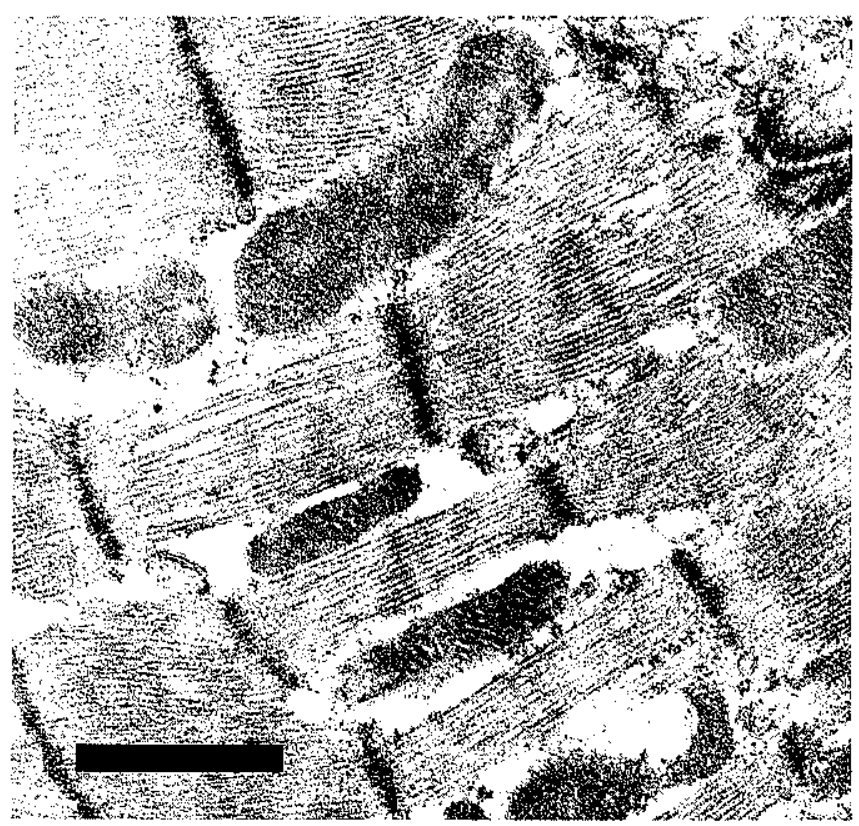

the effect of RR on the myofilaments might differ at different temperatures. In addition, other experimental conditions such as the composition of the immersion solution for the skinned preparation could also explain the different results between the two preparations. Furthermore, some reports have stated a decrease in the $\mathrm{Ca}^{2+}$ sensitivity of the contractile elements due to $\mathrm{RR}[6,12]$, although differing results have also been found [13].

These results indicate that RR influences the myofilaments as well as the SR and mitochondria following RR treatment of intact cardiac muscle for long periods.

Electronmicroscopic study. Intracellular organella are suggested to be stained with RR if $R R$ is applied to the preparation with a fixative $[12,25,26]$. However, there are no morphological reports regard-

Fig. 5. An electronmicrograph of papillary muscies treated with $50 \mu \mathrm{M}$ RR for $80 \mathrm{~min}$ at $30^{\circ} \mathrm{C}$ during the application of ES at $0.2 \mathrm{~Hz}$ and fixed with $2.5 \%$ glutaraldehyde. After glutaraldehyde fixation, the specimen was post-fixed with $1 \% \mathrm{OsO}_{4}$. The submembraneous space of the mitochondrial outer membrane is darkly stained, as indicated by the arrows. Scale bar indicates $1 \mu \mathrm{m}(\mathbf{A})$. The graph shows the incidence (\%) of mitochondria which contains darkly stained outer membrane in the surface and core of the preparation. In the control, no darkly stained membrane was recognized in the surface $(0 / 553)$ or core (0/457) (B).

Fig. 6. An electronmicrograph of papillary muscles treated with $500 \mu \mathrm{M}$ RR for $110 \mathrm{~min}$ at $30^{\circ} \mathrm{C}$ during the application of ES at $\mathbf{0 . 2 ~ H z}$ and fixed with $\mathbf{2 . 5 \%}$ glutaraldehyde. In this case, $\mathrm{OsO}_{4}$ was not used for postfixation. After fixation, one of the two specimens was stained with uranyl acetate and lead citrate (A). The other one was not stained (B). Scale bars indicate $1 \mu \mathrm{m}$. 
ing the permeation of RR through intact cardiac cell membrane.

The present results revealed that darkly stained mitochondrial membranes were found in the specimens treated with RR before fixation with glutaraldehyde and $\mathrm{OsO}_{4}$. If $\mathrm{OsO}_{4}$ is deoxidized by $\mathrm{RR}$, which is a potent reducing agent, the deoxidized $\mathrm{OsO}_{4}$ is recognized as black deposits in the membrane. An electrondense matrix of mitochondria was observed in the RR $(500 \mu \mathrm{M})$-treated specimens fixed with only glutaraldehyde (no $\mathrm{OsO}_{4}$, uranyl acetate, or lead citrate). Therefore, $\mathrm{OsO}_{4}$ and other electron staining agents are not critical for the electron-dense membrane and the matrix of mitochondria. Thus, the darkly stained mitochondrial membrane is not an artifact of the staining method.

The percentage of mitochondria with darkly stained membrane in the surface area was greater than that of the core (Fig. 5B), indicating that RR slowly diffuses from the surface to the core of the intact papillary muscles.

These results clearly show that $R R$ can penetrate through the intact cardiac cell membrane and bind to mitochondria. However, the percentage of darkly stained mitochondrial membrane was about $8 \%$ although no darkly stained mitochondria was exclusively recognized in the control specimen. The question is whether or not the small percentage of the stained mitochondria explains the functional alterations observed in this study. Our explanation is as follows. The darkly stained mitochondria are the most highly affected by RR, and the function of mitochondria is influenced by RR even though ultrastructural change is not observed. For example, $\mathrm{Ca}^{2+}$ release from the SR in electrical stimulation was inhibited by RR but no ultrastructural alterations were recognized in the SR. The sensitivity to detect morphological change, therefore, is probably much lower than that to detect functional alterations. Thus, the parallel changes in the function and ultrastructure are not necessary.

In conclusion, RR permeates through the cell membrane of intact mammalian cardiac muscle and modifies the cellular functions.

We thank Ms. Mary Beth Sibuya for reading the manuscript and Ms. Naoko Tomizawa for technical assistance. This work was supported by a grant from the Japan Women's Medical Society to $E$. T.

\section{REFERENCES}

1. Fletcher JM, Greenfield BF, Hardy CJ, Scargill D, and Woodhead JL: Ruthenium red. J Chem Soc Part II:
2000-2006, 1961

2. Sterling C: Crystal-structure of ruthenium red and stereochemistry of its pectic stain. Am J Bot 57: 172175,1970

3. Luft JH: Ruthenium red and violet. 1. Chemistry, purification, methods of use for electron microscopy and mechanism of action. Anat Rec 171: 347-368, 1971

4. Chamberlain BK, Volpe $P$, and Fleischer $S$ : Inhibition of calcium-induced calcium release from purified cardiac sarcoplasmic reticulum vesicles. J Biot Chem 259: 7547-7553, 1984

5. Antoniu B, Kim DH, Morii M, and Ikemoto N: Inhibitors of $\mathrm{Ca}^{2+}$ release from the isolated sarcoplasmic reticulum. 1. $\mathrm{Ca}^{2+}$ channel blockers. Biochem Biophys Acta 816: 9-17, 1985

6. Volpe P, Salviati $G$, and Chu A: Calcium-gated calcium channels in sarcoplasmic reticulum of rabbit skinned skeletal muscle fibers. J Gen Physiol 87: 289-303, 1986

7. Baylor SM, Hollingworth S, and Marshall MW: Effects of intracellular ruthenium red on excitation-contraction coupling in intact frog skeletal muscle fibres. J Physiol (Lond) 408: 617-635, 1989

8. Mack MM, Zimányi I, and Pessah IN: Discrimination of multiple binding sites for antagonists of the calcium release channel complex of skeletal and cardiac sarcoplasmic reticulum. J Pharmacol Exp Ther 262: 10281037, 1992

9. Vasington FD, Gazzotti P, Tiozzo R, and Carafori E: The effect of ruthenium red on $\mathrm{Ca}^{2+}$ transport and respiration in rat liver mitochondria. Biochim Biophys Acta 256: 43-54, 1972

10. Rossi CS, Vasington FD, and Carafoli E: The effect of ruthenium red on the uptake and release of $\mathrm{Ca}^{2+}$ by mitochondria. Biochem Biophys Res Commun 50: 846852, 1973

11. Reed KC and Bygrave FL: The inhibition of mitochondrial calcium transport by lanthanides and ruthenium red. Biochem J 140: 143-155, 1974

12. Zacharová $D$, Uhrík $B$, Henček $M$, Lipskaja $E$, and Pavelková J: Effects of ruthenium red on excitation and contraction in muscle fibres with $\mathrm{Ca}^{2+}$ electrogenesis. Gen Physiol Biophys 9: 545-568, 1990

13. Zhu $Y$ and Nosek TM: Ruthenium red affects the contractile apparatus but not sarcoplasmic reticulum $\mathrm{Ca}^{2+}$ release of skinned papillary muscle. Pflügers Arch 420: 255-258, 1992

14. Tanaka E, Kawai $M$, Kurihara $S$, Hotta $Y$, and Soji T: Effects of ruthenium red on aequorin-injected ferret ventricular muscles. Jpn J Physiol 45: S58, 1995

15. Sitsapesan R, Montgomery RAP, MacLeod KT, and Williams AJ: Sheep cardiac sarcoplasmic reticulum calcium-release channels: modification of conductance and gating by temperature. J Physiol (Lond) 434: 469488,1991

16. Kurihara S and Sakai T: Effects of rapid cooling on mechanical and electrical responses in ventricular muscle of guinea-pig. J Physiol (Lond) 361: 361-378, 1985

17. Bers DM, Bridge $\mathrm{JHB}$, and Spitzer $\mathrm{KW}$ : Intracellular $\mathrm{Ca}^{2+}$ transients during rapid cooling contractures in guinea-pig ventricular myocytes. J Physiol (Lond) 417: $537-553,1989$ 
18. Tanaka $\mathrm{E}: \mathrm{Ca}^{2+}$ release induced by rapid cooling and caffeine in ferret ventricular muscles. Jpn J Physiol 47: 263-272, 1997

19. Tanaka $E$ and Kurihara S: Contribution of mitochondria to the removal of intracellular $\mathrm{Ca}^{2+}$ induced by caffeine and rapid cooling at low temperatures in ferret ventricular muscles. Jpn J Physiol 47: 251-262, 1997

20. Rousseau E, Smith JS, Henderson JS, and Meissner G: Single channel and ${ }^{45} \mathrm{Ca}^{2+}$ flux measurements of the cardiac sarcoplasmic reticulum calcium channel. Biophys J 50: 1009-1014, 1986

21. Puskin JS, Gunter TE, Gunter KK, and Russell PR: Evidence for more than one $\mathrm{Ca}^{2+}$ transport mechanism in mitochondria. Biochemistry 15: 3834-3842, 1976

22. Jung DW, Davis $\mathrm{MH}$, and Brierley GP: Estimation of the $\mathrm{pH}$ gradient and Donnan potential in de-energized heart mitochondria. Arch Biochem Biophys 263: 19-28, 1988

23. Gunter TE and Pfeiffer DR: Mechanisms by which mitochondria transport calcium. Am J Physiol 258: C755C786, 1990

24. Emerson J, Clarke MJ, Ying W-L, and Sanadi DR: The component of "ruthenium red" responsible for inhibition of mitochondrial calcium ion transport. Spectra, electrochemistry, and aquation kinetics. Crystal structure of $\mu-\mathrm{O}-\left[\left(\mathrm{HCO}_{2}\right)\left(\mathrm{NH}_{3}\right)_{4} \mathrm{Ru}\right]_{2} \mathrm{Cl}_{3}$. J Am Chem Soc 115: 11799-11805, 1993

25. Howell JN: Intracellular binding of ruthenium red in frog skeletal muscle. J Cell Biol 62: 242-247, 1974

26. Luft JH: Ruthenium red and violet. II. Fine structural localization in animal tissues. Anat Rec 171: 369-417, 1971 